\title{
Dynamic spectrum allocation with admission control based on cognitive radio for QoS support in multiple wireless network
}

\author{
Mohammed Raiss-El-Fenni ${ }^{1,4^{*}}$, Rachid El-Azouzi ${ }^{1}$, Mohamed El-Kamili ${ }^{2}$, Khalil Ibrahimi ${ }^{3}$ \\ and EL Houssine Bouyakhf ${ }^{4}$
}

\begin{abstract}
Cognitive radio is an emerging technology in wireless access, aimed at vastly improving the way radio spectrum is utilized. In this article, we investigate the performance improvement gained by applying cognitive radio to a multiple wireless service providers (WSPs). We consider several WSPs and two types of users: primary (licensed) and secondary (unlicensed) users. Two different schemes are proposed for unlicensed users to manage their handoff. These two schemes are different in the sense that one allows to each WSP to give more priority to his own unlicensed users by dropping low priority users, while the other does not allow that. The system is modeled by a Markov process, with continuous time and finite state space. Due to the very large number of states, we propose a robust method to approximate the stationary probability distribution vector of Markov process. Based on this approximation, we develop several performance metrics, blocking and dropping probabilities for both kinds of unlicensed users. Numerical results show that this approximation is very close to the exact solution. Finally, we show that spectrum utilization of cognitive users increases with the proposed schemes especially in medium and high traffic.
\end{abstract}

Keywords: Cognitive radio network, Spectrum utilization, Markov process, Fixed point method

\section{Introduction}

Cognitive radio is a paradigm for wireless communication in which either a WSP or a wireless node changes its transmission or reception parameters to communicate efficiently avoiding interference with licensed or unlicensed users [1]. The term was initially applied to extending software radios with a self awareness about its characteristics and requirements, in order to determine an appropriate radio etiquette to be used $[2,3]$. This alteration of parameters is based on the active monitoring of several factors in the external and internal radio environment, such as radio frequency spectrum, user behavior and network state. In a network supporting cognitive radio, secondary users (SUs) opportunistically use the spectrum that is normally assigned to primary users (PUs) but not being used at

\footnotetext{
*Correspondence: mohammed.raiss@etd.univ-avignon.fr

1 LIA/CERI, University of Avignon, Agroparc BP 1228, Avignon, France

4 LIMIARF/FSR, University of Mohammed V-Agdal, BP 1014 RP, Rabat, Morocco

Full list of author information is available at the end of the article
}

a particular time and geographic location. Thus, a secondary user must leave the channel once detecting the presence of a licensed user. At the same time, the SU will scan all other channels and handoff to another unused one if available; otherwise, its communication is interrupted. Once interrupted, the SU can either leave the system or wait in a queue so that its connection is terminated or suspended. Some concerns on unlicensed spectrum usage and performance analysis of cognitive radio networks are discussed in $[4,5]$.

In [4], Zhang propose a new dynamic spectrum access scheme for cognitive radio wireless networks with and without buffering for secondary users to avoid direct blocking. A Markov model was developed to analyze the proposed spectrum sharing policies. The result indicates that the buffer is able to significantly reduce the SU blocking probability and noncompletion probability with very minor increased forced termination probability. The analytic model has been verified by simulation. A rigorous study of performance in opportunistic spectrum access systems that limit disruptions to unpredictable primary 
users is presented in [6]. The goal was to understand whether dynamic spectrum access can provide reliable spectrum to secondary users while respecting hard disruption limits that protect primary-user transmissions.

Zhang [7] consider that in a cognitive radio network (CRN), instead of direct leaving, an interrupted secondary user is suspended to wait for accessing another channel in a call level queue. The policy for handling the packets generated by the secondary user during the suspending period can be either delaying or discarding. According to these two packet level policies, the queue is separated into two parts, delay queue and discard queue. The article mainly analyzes the performance of a CRN with such a two part call level queue. Other studies were interested in economical aspect. In [8], a Stackelberg game between three players; spectrum owner, primary users and secondary users is presented under the opportunistic spectrum access (OSA) model, where the secondary users (followers) share the channel with primary users in time and SUs access is performed through a non perfect listenbefore-send scheme. It is shown through simulations that the spectrum owner can enhance its revenue by allowing OSA with a non zero interference probability to the primary users. Virtual unlicensed spectrum is another way to share bands with primary users without interferences. Thus, in [5] a cognitive radio approach for usage of virtual unlicensed spectrum is presented. A vision of a cognitive radio based approach that uses allocated spectrum in an opportunistic manner to create virtual unlicensed bands, i.e., bands that are shared with the primary users on a non-interfering basis. Dynamic spectrum management techniques are used to adapt to immediate local spectrum availability. The authors define the system requirements for this approach, as well as the general architecture and basic physical and link layer functions. In general, there are two basic operation models for SUs: OSA which is also called the spectrum overlay paradigm versus spectrum sharing (SS) or the spectrum underlay paradigm. In the OSA model, the SUs are allowed to transmit over the band of interest when all the PUs are not transmitting at this band. One essential enabling technique for OSA based SUs is spectrum sensing; where the SUs individually or collaboratively detect active PU transmissions over the band, and decide to transmit if the sensing results indicate that all the PU transmitters are inactive at this band with a high probability. The key operation is spectrum sensing; the interested readers may refer to, e.g., [9-12] for an overview of the state of art results in this area. As a counterpart, the SS model allows the SUs to transmit simultaneously with PUs at the same band even if they are active, provided that the SUs know how to control their resultant interference [13]. For instance, in [14] packet collision probability was considered as the PU protection requirement. Under this requirement, the SU must guarantee that the packet collision probability of a PU packet is less than a certain threshold specified by the PU.

In this article, we consider a system with multiple wireless service providers (WSPs) where multiple SUs are allowed to access the unused licensed spectrum bands without conflicting with PUs. In order to protect the QoS of PUs, each WSP reserves a number of channels allowed to them. Their access to these channels should not be affected by the SUs. We assume that the SUs are equipped with cognitive radio equipment and are able to detect the presence of the licensed users [15,16]. In [17], Ishibashi et al. were focused on similar problem by studying the benefits that can be gained by adding cognitive radio capabilities to a system of PUs. They considered the case where all SUs are in the same level of priority. The symmetric case was studied, i.e., the case when all networks in the system have the same capacities and number of channels. Moreover, in their model they did not explicit the interaction between networks (handoff of cognitive calls) in terms of migration rates $[18,19]$. Also, the transition probability matrix and the stationary distribution were solved only based on simulations.

In this article, we consider a general framework that extends all limitation of the above study. Furthermore, we consider the prioritization among the SUs while accessing the channel. We distinguished two schemes as follows:

- No priority scheme: The agreement between the WSP and the primary users is to guarantee a certain QoS in term of interferences and availability of channels, like throughput and blocking probability. In order to assure the QoS for the licensed users, the WSP determine a threshold on the number of channels allowed to licensed users and reserves the rest of bandwidth to unlicensed users. Since the SU are capable to detect the presence of the PUs, it is allowed to dynamically access unused channels in the primary user bandwidth. Another alternative for SU, is to handoff to another WSP belonging to the system if it has some available resources. Even if the threshold will restrict the use of some channels to PUs, it will not affect their QoS. Indeed, the threshold is set by the WSP based on its statistics about the arrival and service time of PUs.

- Priority scheme: In this scheme we introduce the priority among SUs in the above scheme. In particular, we assume that a SU has more priority on its home WSP than other SU belonging to another WSP. However, a foreign SU may be pushed out from a WSP if it is fully occupied and reclaim those resources for its own use.

In practice, there is no reserved bandwidth to SUs, and the threshold defined for PUs is exactly the total number 
of available channels. Therefore, SUs use only empty channels when the PUs are absent or inactive. This case is covered by our article. The interest of this work is to study the benefit of including such threshold by WSPs in term of performance while insuring a QoS to PUs all the time. Indeed, a user can use licensed channels for real time calls in order to guarantee the desired QoS, while the best effort calls are performing using the unlicensed channels with cognitive equipment. Reducing the total cost of the service could be another reason of such kind of priority.

We called SUs in their home WSP, own SUs and for others foreign SUs. We define a priority among SUs in each WSP. This priority means that an own SU can drop a foreigner one, under some conditions as described in the following section.

Summing up, the main contributions of the article are:

- We propose a new model to describe and analyze the interaction between WSPs with infrastructure deployed in the same geographical area and cover a shared pool of end users. We model the dynamic system as a Markov chain, and derive the performances of the WSPs under two schemes. Due to very large number of states, we propose a new method based on the fixed point to approximate the stationary distribution. Numerical results show that this approximation is very close to the exact solution.

- Unlike several studies, our model introduces the priority among secondary users and addresses the issue of spectrum handoff under prioritized SU calls. The performance of the two schemes are evaluated in term of blocking and dropping probabilities.

- We apply a threshold to PUs while insuring their QoS. We study the impact of this threshold and how it influence the spectrum efficiency. Finally, we perform an extensive numerical analysis to provide insightful results about the spectrum utilization of cognitive users. Results show that the spectrum utilization increases with the proposed schemes especially in high load traffic.

The remainder of the article is organized as follows. In the following section, we formulate the problem and describe the model that we use. After that we develop an analysis model with respect to the no priority scheme. Followed by the model that gives more priority to own SU calls than foreign calls in each WSP. Next, we derive and evaluate the performance of each scheme and finally, in the last section, we give some numerical results and end with a concluding summary.

\section{System model}

The system under consideration is a collection of $N$ wireless services providers (WSPs) with infrastructure deployed in the same geographical area and cover a shared pool of end user (see the Figure 1 with three service providers). The WSP $i$ possesses $K_{i}$ channels and without loss of generality each user requires the capacity of one channel for the duration of its service in order to fulfil its requirements. We consider three types of users:

- The PUs related to a specific WSP with a guaranteed QoS, dedicated channels and without the possibility to have service in other WSPs.

- The users who choose to split their traffic into two different parts to benefit from the advantages of both licensed and unlicensed channels.

- The last kind is SUs who are not attached to any WSP and sense all channels of the system looking for spectrum holes.

A perfect channel is assumed in which a channel is either busy or idle. It is also assumed that radio systems always detect radio resource allocations of each others, here the radio resource refers to bandwidth. We assume that spectral scanning is performed instantaneously, so there is no scanning delay. We assume that PUs arrivals of WSP $i$, follow a Poisson distribution with rate $\lambda_{i}^{\mathrm{PU}}$ and their service time is exponentially distributed with mean $1 / \mu_{i}^{\mathrm{PU}}$. We define $\rho_{\mathrm{PU}}^{i}=\lambda_{\mathrm{PU}}^{i} / \mu_{\mathrm{PU}}^{i}$. Since the PUs are not affected by the SUs, the probability to have $n_{\mathrm{PU}}^{i}$ PUs in the WSP $i$ is given by

$$
q_{n_{\mathrm{PU}}}^{i}=\frac{1}{G} \frac{\left(\rho_{\mathrm{PU}}^{i}\right)^{n_{\mathrm{PU}}^{i}}}{n_{\mathrm{PU}}^{i} !}
$$

where $G$ is the normalizing constant. Based on the stationary probability, each WSP $i$ is able to estimate the number of licensed channels in order to evaluate the QoS for primary users. However, if the spectrum of WSP $i$ is not fully utilized, the provider may reserve a part of bandwidth for SUs.

The system considered has a total capacity of $M=$ $\sum_{i=1}^{N} K_{i}$. However, the WSPs belonging to the system are completely independent of each other, with primary users only being served by their home WSP. As a result, when a WSP reaches its capacity, it must start blocking calls, as it has no available channels.

For SUs, if resources are not available on its home WSP, the SU can switch to another WSP $j(j \neq i)$. For both schemes presented in the following sections, a SU may be pushed out if it use a licensed channel and no resource is available among licensed channels. However, our model still cover the classical scenario of cognitive radio where the PUs have the right to use all channels and SUs take advantage of spectrum only when PUs are absent. The threshold was included in 


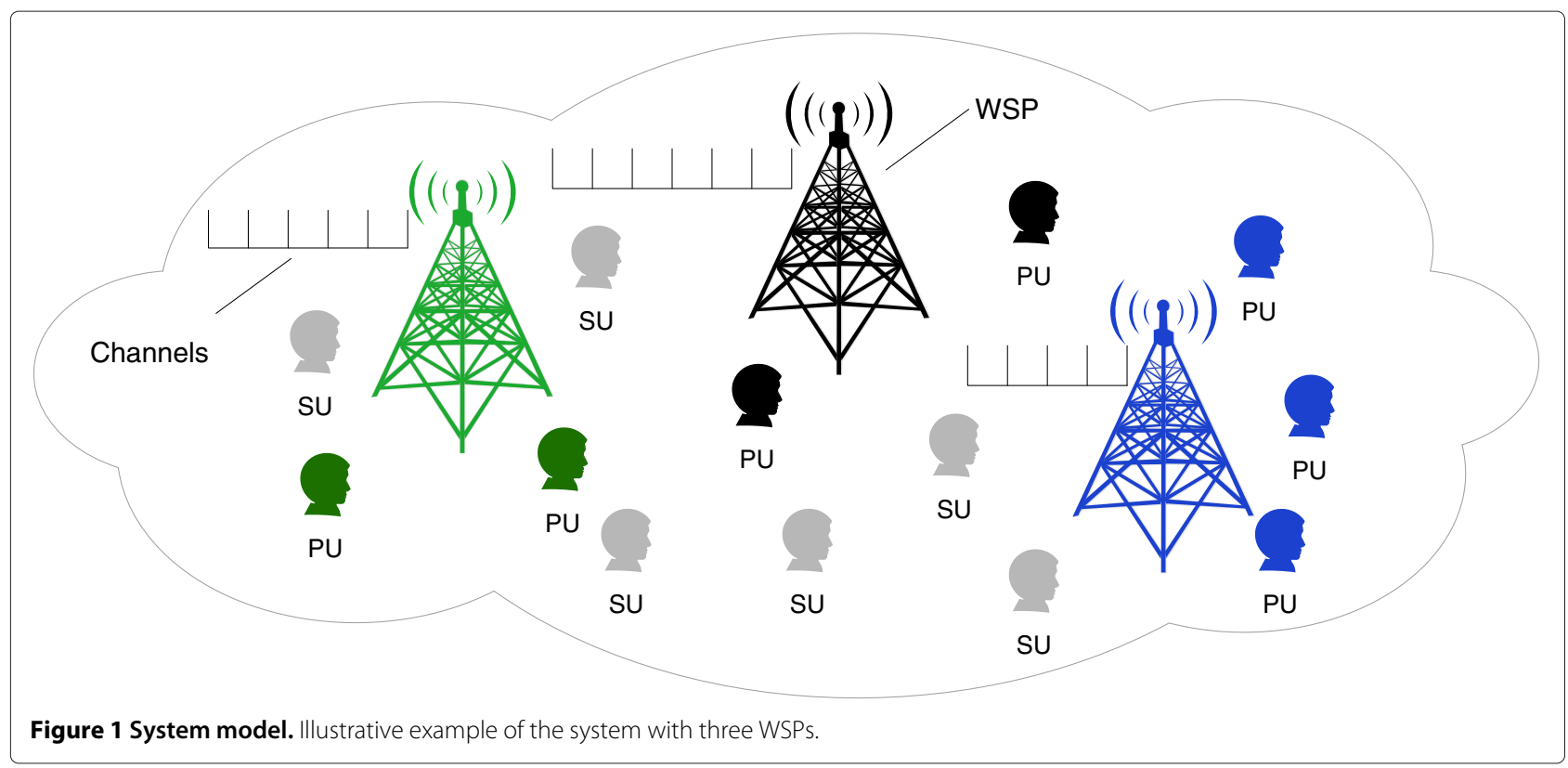

order to study how our scenario may improve the performance under the constraint to guarantee the QoS required by the PUs. Furthermore, our study propose two schemes: These two schemes are different in the sense that one allows to each WSP to give more priority to his own unlicensed users by dropping low priority users, while the other does not allow that. We summarize the main parameters used in this article in Table 1.

\section{Model: no priority scheme}

In this scheme, there is no priority among SUs in the system. Hence, a SU can be dropped from WSP $i$ if a new

\section{Table 1 Main definition parameters}

\begin{tabular}{ll}
\hline Parameter & Definition \\
\hline$M$ & Capacity of the system \\
$N$ & Number of wireless service providers \\
$K_{i}$ & Number of channels in WSP $i$ \\
$T h_{i}$ & Threshold of channels reserved to by WSP $i$ \\
$\mu_{\mathrm{PU}}$ & Service rate of PUs \\
$\mu_{\mathrm{SU}}$ & Service rate of SUs \\
$\lambda_{\text {new,SU }}^{i}$ & Fixed SU arrival rate in WSP $i$ \\
$\lambda_{\text {out, SU }}^{i}$ & External SU arrival rate in WSP $i$ \\
$\lambda_{\mathrm{PU}}^{i}$ & PUs arrival rate in WSP $i$ \\
$\lambda^{j, i}$ & Migration rate from WSP $j$ to $i$ \\
$n_{\mathrm{PU}}^{i}$ & Number of PUs in WSP $i$ \\
$n(i)$ & Total number of users in WSP $i$ \\
$n_{\mathrm{SU}}^{i}$ & Number of SUs in WSP $i$ \\
$n_{\mathrm{SU}}^{-i}$ & Number of external SUs in WSP $i$ \\
\hline
\end{tabular}

arrival call of PU find all licensed channel occupied and the number of PUs in their home, doesn't reach the threshold, i.e., $n_{\mathrm{PU}}^{i}<T h_{i}$, where $n_{\mathrm{PU}}^{i}$ and $T h_{i}$ are, respectively, the total number of PUs and the number of channels allowed for PUs in WSP $i$. A new arrival of PU (resp. SU) will be blocked if $n_{\mathrm{PU}}^{i}=T h_{i}$ (resp. $n_{\mathrm{PU}}^{i}+n_{\mathrm{SU}}^{i}=K_{i}$ ), where $n_{\mathrm{S} U}^{i}$ is the total number of SUs (owner or foreigner) in WSP $i$.

\section{System state and transition rates}

In order to analyze the behavior of the WSPs, the state of the system at time $t$ is defined by

$$
\vec{n}(t)=\left(n_{\mathrm{PU}}^{1}(t), \ldots, n_{\mathrm{PU}}^{N}(t), n_{\mathrm{SU}}^{1}(t), \ldots, n_{\mathrm{SU}}^{N}(t)\right)
$$

then, we model the process $\{\vec{n}(t), t>0\}$ as $2 N$ dimensions quasi birth and death Markov chain with continuous time. Let $n(i)=n_{\mathrm{PU}}^{i}+n_{\mathrm{SU}}^{i}$ be the total number of users in WSP $i$. Thus, we define the space of admissible states as

$$
E=\left\{\vec{n} \in \mathbf{N}^{2 N} \mid n(i) \leq K_{i} ; i=1, \ldots, N\right\} .
$$

Let $\lambda_{\text {new,SU }}^{i}$ be the arrival rate of SUs in WSP $i$ (i.e., users splitting their traffic), and let $\lambda_{\text {out,SU }}$ be the rate of foreign SUs. We assume that this two rates are characterized by a set of poisson process. Also, we assume that the service time distribution is exponential with $\mu_{\mathrm{SU}}^{i}$. The Poisson process has been found to provide a good representation for arrival calls and service time of a call while Internet traffic exhibits a correlation structure over a wide span of time scales. We will analyze the performance of our schemes in which packets arrivals are modeled 
by a Markovian arrival which captures the correlation of inter-arrival times among primary users, among secondary users, as well as between the two types of users. These issues are beyond the scope of this article and will be treated in future study. The transition rates from a state $\vec{n}$ to another state $\vec{n}^{\prime}$ are described as follows. Let $\vec{n}_{i+}^{c}$ be the state corresponding to a new arrival belonging to class c (PU or SU) and we denote this transition by $q_{\left\{\vec{n}_{,} \vec{n}_{i+}^{c}\right\}}$. Let $\vec{n}_{i-}^{c}$ be the state when a call from class $c$ ends successfully its service and leaves the system, and we denote this transition by $q_{\left\{\vec{n}, \vec{n}_{i-}^{c}\right\}}$, and let $\vec{n}_{i+, j-}^{c, c^{\prime}}$ be the state when a new arrival call from class $c$ gives rise to an interrupted call from class $c^{\prime}$, and we denote this transition by $q_{\left\{\vec{n}, \vec{n}_{i+, j-}^{c, c^{\prime}}\right\}}$. Then we have

$$
\begin{aligned}
q_{\left\{\vec{n}, \vec{n}_{i-}^{\mathrm{PU}}\right\}} & =n_{\mathrm{PU}}^{i} \cdot \mu_{\mathrm{PU}}^{i}, \\
q_{\left\{\vec{n}, \vec{n}_{i-}^{\mathrm{SU}}\right\}} & =n_{\mathrm{SU}}^{i} \cdot \mu_{\mathrm{SU}}^{i}, \\
q_{\left\{\vec{n}, \vec{n}_{i+}^{\mathrm{PU}}\right\}} & =\lambda_{\mathrm{PU}}^{i} \cdot \mathbf{1}_{\left\{n_{\mathrm{PU}}^{i} \neq 0\right\}}, \\
q_{\left\{\vec{n}, \vec{n}_{i+}^{\mathrm{SU}}\right\}} & =\lambda_{\mathrm{SU}}^{i} \cdot \mathbf{1}_{\left\{n_{\mathrm{SU}}^{i} \neq 0\right\}}, \\
q_{\left\{\vec{n}_{,} \vec{n}_{i+, j-}^{P U, S U}\right\}} & =\lambda_{\mathrm{PU}}^{i} \cdot
\end{aligned}
$$

Now, the transition probability matrix $\mathbf{Q}$ is derived for the process $\{\vec{n}(t), t>0\}$. The transitions are found for both primary and cognitive calls. The transition probability matrix $\mathbf{Q}=\left[q_{\left(\vec{n}, \vec{n}^{\prime}\right)}\right]$ can be derived. The steady state vector $\pi(\pi=\{\pi(\vec{n}), \vec{n} \in E\})$ of probabilities $\pi(\vec{n})$ can be obtained by resolving the following system:

$$
\pi \cdot \mathbf{Q}=\pi \text { and } \sum_{\vec{n} \in E} \pi(\vec{n})=1
$$

To solve this problem we propose two different ways, the exact solution, by constructing the matrix $Q$ from all feasible states from the overall system. This solution is complicated and takes a very long time to be solved because of the huge number of states present in the space $E$. Also, most of the time, a service provider doesn't have complete or enough information about statistics and parameters used by other service providers, like the number of PUs or the threshold reserved for them. In spite of this problem, Ishibashi et al. [17], solve (2) by simulation without taking in account interactions (migration of SU calls) occurring among the WSPs. In this study, we present a robust approximation to solve the system of linear equations (2), by considering the probability that the total system in state $\vec{n}$ is written as a product of steady state probability of each WSP. In this way we reduce the complexity of the problem and we switch from the study of the space $E$ and the overall system to a smaller space related to each WSP $i$. The interaction among all these WSPs is studied by considering an approximation to cognitive arrival rate in each WSP. This arrival rate depends on the global state $(\vec{n} \in E)$ of the system. Hence, the SU calls arrival rate in the WSP $i$ is given by

$$
\lambda_{\mathrm{SU}}^{i}(\vec{n})=\lambda_{\text {new }, \mathrm{SU}}^{i}+\sum_{j \neq i} \lambda_{\mathrm{SU}}^{j, i}+\lambda_{\text {out }, \mathrm{SU}}^{i}, \quad \text { if } n(i)<K_{i},
$$

where $\lambda_{\mathrm{SU}}^{j, i}$ is the SU call migration rate from WSP $j$ to the WSP $i(j \neq i)$ and $\lambda_{\text {out,SU }}^{i}$ represents the arrival rate of SUs to WSP $i$. The arrival rates $\lambda_{\mathrm{SU}}^{j, i}$ and $\lambda_{\text {out,SU }}^{i}$ are unknown and depends on the random number of SU calls of the WSP $j$ that go elsewhere. These rates depends on the stationary distribution. We give explicit fixed point method to calculate these arrival rates in the following section.

For WSP $i$, we define the sub state space as follows

$$
E_{i}=\left\{\vec{n}_{i} \in \mathbf{N}^{2} \mid n(i) \leq K_{i} ; i=1, \ldots, N\right\},
$$

where $\vec{n}_{i}=\left(n_{\mathrm{PU}}^{i}, n_{\mathrm{SU}}^{i}\right)$. The steady state probability of each WSP $i$ is defined by

$$
\pi_{i}=\left\{\pi_{i}\left(\overrightarrow{n_{i}}\right) \mid \overrightarrow{n_{i}} \in E_{i}\right\}
$$

this steady state probability is given by solving the set of balance equations [20]. The different states are described as follows:

1. The states satisfying $n_{\mathrm{PU}}^{i}+n_{\mathrm{SU}}^{i}<K_{i}$ and $n_{\mathrm{PU}}^{i}<T h_{i}$ (no saturation case).

2. The states satisfying $n_{\mathrm{PU}}^{i}+n_{\mathrm{SU}}^{i}=K_{i}$ and $n_{\mathrm{PU}}^{i}<$ $T h_{i}$, in this case a new $P U$ arrival gives rise to an interrupted $S U$ call.

3. The states satisfying $n_{\mathrm{PU}}^{i}+n_{\mathrm{SU}}^{i}=K_{i}$ and $n_{\mathrm{PU}}^{i}=T h_{i}$, here the request of a new $P U$ or $S U$ arrival is refused by the WSP and they are blocked.

In addition, the summation of all steady state probabilities satisfies the normalization constraint $\sum_{\vec{n}_{i} \in E_{i}} \pi_{i}\left(\overrightarrow{n_{i}}\right)=1$. Combining the balance equations, we can solve the steady state probability distribution. Therefore, the transit rate matrix $\mathbf{Q}_{i}$ and the solution of linear equations $\pi_{i}=\pi_{i} \cdot \mathbf{Q}_{i}$, can be obtained. The set of linear equations can be solved by using an iterative method SOR [20].

\section{Calculation of migration rates}

A SU call of WSP $j$ go elsewhere if $n(j)=K_{j}$ (i.e., WSP $j$ uses all of its own resources). Let $a(\vec{n})$ be the number of WSPs that still have resources at the state $\vec{n}$, then we have

$$
a(\vec{n})=\sum_{i=1}^{N} \mathbf{1}_{\left\{n(i)<K_{i}\right\}}
$$


The probability $(\pi(\vec{n}))$ that the system is in the state $\vec{n}$, is given by

$$
\pi(\vec{n})=\frac{1}{\alpha} \Pi_{i=1}^{N} \pi_{i}\left(\overrightarrow{n_{i}}\right)
$$

where $\alpha=\sum_{\vec{n} \in E} \Pi_{i=1}^{N} \pi_{i}\left(\vec{n}_{i}\right)$. Then, the SU call arrival rate from WSP $j$ to WSP $i$ is given by

$$
\lambda_{\mathrm{SU}}^{j, i}=\sum_{\vec{n} / n(j)=K_{j}, n(i)<K_{i}} \frac{\pi(\vec{n})}{a(\vec{n})} \lambda_{\text {new }, \mathrm{SU}}^{j} .
$$

Also the arrival rate of a cognitive user $\lambda_{\text {out,SU }}^{i}$ is given by

$$
\lambda_{\text {out }, \mathrm{SU}}^{i}=\sum_{\vec{n} \in E, n(i)<K_{i}} \frac{\pi(\vec{n})}{a(\vec{n})} \lambda_{\text {out,SU }} .
$$

In order to obtain the arrival rate $\lambda_{\mathrm{SU}}^{i}(\vec{n})$ of SU call arrival to WSP $i$ from (3), we first need to compute the SU call arrival rate from $j$ to WSP $i\left(\lambda_{\mathrm{SU}}^{j, i}, j \neq i\right)$. To do this we follow the Migration rate computing algorithm. With the same way we compute $\lambda_{\text {out,SU} \text {. }}^{i}$

\section{Migration rate computing algorithm:}

1. Initialize the migration rates by zero in $(3): \lambda_{\text {old, SU }}^{j, i}=0$.

2. Calculate the steady state probability $\pi(\vec{n})$ from (4).

3. Derive the new values of migration rates from (5) and denoted by $\lambda_{\text {new,SU}}^{j, i}$.

4. Check the convergence of the migration rates between old and new rates, i.e., if $\left|\lambda_{\text {new,SU }}^{j, i}-\lambda_{\text {old,SU }}^{j, i}\right|<$ $\xi$, where $\xi$ is a very small positive number, then the new migration rates will be used to compute the performance metrics. Otherwise, go to step 2 with the new migration rates as initial values. The iterations are continued until to reach the convergence of rates.

Since the Markov chain has a stationary distribution $\pi$, a migration rate $\lambda_{\mathrm{SU}}^{j, i}$ exist and can be estimated by the previous Algorithm.

\section{Model: priority scheme}

In this section, we distinguished among own and foreign SUs in a WSP. We give more priority to the users that choose to split their traffic and send a part from it without using their dedicated channels among foreign SUs. This choice of users can be motivated by the nature of their operations (audio call, file transfer ...). Based on this priority, if all resources are occupied in his own WSP, a new SU will check if a foreign SU use one of the channels, and ask for dropping him out. As a result, an SU will not be dropped only by PUs as in the No priority scheme, but also by other SUs if the conditions discussed above are satisfied.

\section{System state and transition rates}

As described in the first scheme, we define the system state at time $t$ as follows

$$
\vec{n}(t)=\left(n_{\mathrm{PU}}^{i}(t), n_{\mathrm{SU}}^{i}(t), n_{\mathrm{SU}}^{-i}(t), i=1, \ldots, N\right),
$$

where $n_{\mathrm{SU}}^{-i}(t)$ is the number of SUs of other WSPs in the WSP $i$. Then, we model the process $\{\vec{n}(t), t>0\}$ as $N(N+1)$ dimensions quasi birth and death Markov chain with continuous time and finite state space.

Let $n(i)=n_{\mathrm{PU}}^{i}+n_{\mathrm{SU}}^{i, i}+n_{\mathrm{SU}}^{-i}$ be the total number of users in WSP $i$, where $n_{\mathrm{SU}}^{i, i}$ represents the number of own SUs. Thus, we define the space of the admissible states as follows

$$
F=\left\{\vec{n} \in \mathbf{N}^{N(N+1)} \mid n(i) \leq K_{i} ; i=1, \ldots, N\right\} .
$$

As in the first scheme, arrival and service rates of PUs are characterized by a set of Poisson processes with parameter $\lambda_{\mathrm{PU}}^{i}$ and an exponential distribution with parameter $\mu_{\mathrm{PU}}^{i}$, respectively. We assume that the service time of SUs in WSP $i$ is distributed exponentially with parameter $\mu_{\mathrm{SU}}^{i}$. The arrival rate of SUs depends on the global state $(\vec{n} \in F)$ of the system. The arrival rate of SUs in WSP $i$ is given by

$$
\lambda_{\mathrm{SU}}^{i}(\vec{n})=\lambda_{\text {new }, \mathrm{SU}}^{i}+\sum_{j \neq i} \lambda_{\mathrm{SU}}^{j, i}+\lambda_{\text {out,SU }}^{i}, \quad \text { if } n(i)<K_{i} .
$$

As indicated in the first scheme, the matrix $Q$ of transitions is obtained over the feasible state space $F$. Then, we derive a new system of equations like (2) and find the global steady state denoted by $\bar{\pi}(\bar{\pi}=\{\bar{\pi}(\vec{n}), \vec{n} \in F\})$. We solve the problem in two ways, by constructing the above matrix $\boldsymbol{Q}$ and by the approximative method, to derive the steady state probabilities.

To obtain the matrix $Q$, let us define a new class $c$ by $S U^{-}$, that represents the foreign SUs. In addition to the transitions defined in (1), we have

$$
\begin{aligned}
q_{\left\{\vec{n}_{,} \vec{n}_{i-}^{S U-}\right\}} & =n_{\mathrm{SU}}^{-i} \cdot \mu_{\mathrm{SU}}^{i}, \\
q_{\left\{\vec{n}, \vec{n}_{i+}^{S U-}\right\}} & =\lambda_{\mathrm{SU}}^{-i} \cdot \mathbf{1}_{\left\{n_{\mathrm{PU}}^{-i} \neq 0\right\}}, \\
q_{\left\{\vec{n}, \vec{n}_{i+, j-}^{P U, S{ }^{-}}\right\}} & =\lambda_{\mathrm{PU}}^{i}, \\
q_{\left\{\vec{n}, \vec{n}_{i+, j-}^{S U, S U^{-}}\right\}} & =\lambda_{\mathrm{SU}}^{i} .
\end{aligned}
$$


For the approximative solution we define the sub state space for WSP $i$ as follows

$$
F_{i}=\left\{\vec{n}_{i} \in \mathbf{N}^{3} \mid n(i) \leq K_{i} ; i=1, \ldots, N\right\},
$$

where $\overrightarrow{n_{i}}=\left(n_{\mathrm{PU}}^{i}, n_{\mathrm{SU}}^{i}, n_{\mathrm{SU}}^{-i}\right)$. The steady state probability of WSP $i$ is defined by

$$
\bar{\pi}_{i}=\left\{\bar{\pi}_{i}\left(\overrightarrow{n_{i}}\right) \mid \overrightarrow{n_{i}} \in F_{i}\right\}
$$

This steady state probability is given by solving the set of balance equations [20]. We distinguish four cases:

1. The states satisfying $n(i)<K_{i}, n_{\mathrm{PU}}^{i}<T h_{i}$ and $n_{\mathrm{SU}}^{-i} \neq$ 0 and it represents the non saturated states.

2. If we have states satisfying $n(i)=K_{i}$ and $n_{\mathrm{PU}}^{i}<T h_{i}$, the arrival of a new PU gives rise to an interrupted SU. If a foreign $\mathrm{SU}$ occupies a channel, he is dropped first, if it's not the case, an own SU is dropped.

3. If the states satisfy the conditions $n(i)=K_{i}$ and $n_{\mathrm{SU}}^{-i} \neq 0$, the arrival of a new own SU gives rise to an interrupted external SU.

4. If the states satisfy the conditions $n(i)=K_{i}, n_{\mathrm{PU}}^{i}<$ $T h_{i}$ and $n_{\mathrm{SU}}^{-i}=0$, the arrival of a new PU gives rise to an interrupted own SU.

The summation of all steady state probabilities satisfies the normalization constraint $\sum_{\vec{n}_{i} \in F_{i}} \bar{\pi}_{i}\left(\vec{n}_{i}\right)=1$. We solve the set of the linear equations and consequently the steady state probability distribution by using the SOR [20] method.

\section{Calculation of migration rates}

A SU from WSP $j$ decides to handoff to another WSP if $n(j)=K_{j}$ and $n_{\mathrm{SU}}^{-j}=0$ (i.e., WSP $j$ uses all its own resources without the presence of foreign SUs). Then, the arrival rate (migration) of SUs from WSP $j$ to $i$ is given by

$$
\lambda_{\mathrm{SU}}^{j, i}=\sum_{\vec{n} / n(j)=K, n(i)<K_{i}, n_{\mathrm{SU}}^{-j}=0} \frac{\bar{\pi}(\vec{n})}{a(\vec{n})} \lambda_{\text {new,SU }}^{j},
$$

where

$$
\bar{\pi}(\vec{n})=\frac{1}{\beta} \Pi_{i=1}^{N} \bar{\pi}_{i}\left(\vec{n}_{i}\right),
$$

and $\beta=\sum_{\vec{n} \in F} \prod_{i=1}^{N} \bar{\pi}_{i}\left(\vec{n}_{i}\right)$. In order to obtain the arrival rate $\lambda_{\mathrm{SU}}^{i}(\vec{n})$ from (7), we need first to compute $\left(\lambda_{\mathrm{SU}}^{j, i}, j \neq i\right)$. To do this we apply again the Migration rate computing algorithm defined previously by replacing the Equations (3), (4), and (5) by these analogous (7), (10), and (9), respectively. Note that the arrival rate $\lambda_{\text {out,SU }}^{i}$ has the same value as in the previous scheme.

\section{Performance evaluation}

We have used three classic QoS metrics to evaluate the performance of the system: blocking probability, dropping probability and spectrum utilization metric. Each is calculated based on the state and transition probabilities for the two schemes as follows:

\section{Blocking probabilities}

Let $P_{B}(i, P U)$ be the blocking probability for PUs calls belonging to WSP $i$. A PU is blocked if he arrives while its home WSP is already using all its available resources dedicated to PUs (i.e., $n_{\mathrm{PU}}^{i}+1>T h_{i}$ ). Therefore, the probability of blocking for the two schemes is expressed as follows:

$$
P_{B}(i, P U)=\sum_{\vec{n} \in E} \pi(\vec{n}) \cdot \mathbf{1}_{\left\{n_{\mathrm{PU}}^{i}+1>T h_{i}\right\}} .
$$

Similarly, $P_{B}^{1}(S U)\left(\right.$ resp. $\left.P_{B}^{2}(i, S U)\right)$ are the SUs blocking probabilities for the No priority scheme (resp. for the priority scheme). However, the conditions for blocking are different depending on the scheme. Indeed, in order to be blocked many conditions must be satisfied when the a SU arrives:

- A new SU in the no priority scheme is blocked if all resources in all WSPs are occupied $\left(\sum_{k=1}^{N} n(k)=M\right)$. Therefore, the blocking probability is

$$
P_{B}^{1}(S U)=\sum_{\vec{n} \in E} \pi(\vec{n}) \cdot \mathbf{1}_{\left\{\sum_{k=1}^{N} n(k)=M\right\}} .
$$

- A new SU using the priority scheme is blocked if all resources in all WSPs are occupied and the own WSP of this SU is fully occupied by its own users $\left(n_{\mathrm{SU}}^{-i}=0\right)$. Then the blocking probability is

$$
P_{B}^{2}(i, S U)=\sum_{\vec{n} \in F} \bar{\pi}(\vec{n}) \cdot \mathbf{1}_{\left\{\sum_{K=1}^{N} n(k)=M, n_{\mathrm{SU}}^{-i}=0\right\}} .
$$

\section{Dropping probabilities}

Once service begins, the primary connections are never interrupted or dropped for the two schemes (i.e., $P_{D}^{1}(i, P U)=P_{D}^{2}(i, P U)=0$ ). Therefore, only SUs (own and foreigner) can be dropped. Concerning the priority scheme, a SU using a channel in WSP $i$ is dropped only if a new PU arrive and the condition $n_{\mathrm{PU}}^{i}<T h_{i}$ occurs. Then the dropping probability is given by

$$
P_{D}^{1}(i, S U)=\sum_{\vec{n} \in E} \pi(\vec{n}) \cdot \mathbf{1}_{\left\{n(i)=K_{i}\right\}} \cdot \mathbf{1}_{\left\{n_{\mathrm{PU}}^{i}<T h_{i}\right\}} .
$$


As for the priority scheme a SU in his own WSP $i$ is dropped if a new PU arrive and the two conditions $n_{\mathrm{PU}}^{i}<$ $T h_{i}$ and $n_{\mathrm{SU}}^{-i}=0$ occur. Then the dropping probability is given by

$$
P_{D}^{2}(i, S U)=\sum_{\vec{n} \in F} \bar{\pi}(\vec{n}) \cdot \mathbf{1}_{\left\{n(i)=K_{i}\right\}} . \mathbf{1}_{\left\{n_{\mathrm{PU}}^{i}<T h_{i}\right\}} \cdot \mathbf{1}_{\left\{n_{\mathrm{SU}}^{-i}=0\right\}} .
$$

Several conditions must occur in order to drop a foreigner SU when a new arrival event to WSP $i$ happens. These conditions differ slightly, depending on whether the incoming user is a primary or a secondary. In both cases, these two conditions must occur:

- All the $M$ channels in the system are occupied $\sum_{k=1}^{N} n(k)=M .(i)$

- WSP $j$ is using all resources that it physically possesses by its own users (i.e., no external SUs). Formally we have $n(j)=K_{j}$ and $n_{\mathrm{SU}}^{-j}=0$. (ii)

If the user that arrives is primary, the following condition (iii) must also be true:

- WSP $i$ uses less resources than its specified threshold $\left(n_{\mathrm{PU}}^{i}<T h_{i}\right) .(i i i)$

Whereas if the user that arrives is cognitive, condition (iv) must occurs:

- WSP $i$ uses less resources than its physical capacity $\left(n_{\mathrm{SU}}^{-i} \neq 0\right)$. (iv)

Summing up all the results implies that the dropping probability in a WSP $i$ of a SU making a handover from WSP $j$ by a another SU from $i, i \neq j$ is given by

$$
\begin{aligned}
P_{D}^{2}(j, i, S U)= & \left(\sum_{\vec{n} \in F} \bar{\pi}(\vec{n}) \mathbf{1}_{\left\{n_{\mathrm{PU}}^{i}<T h_{i}\right\}}\right. \\
& \left.+\sum_{\vec{n} \in F} \bar{\pi}(\vec{n}) \mathbf{1}_{\left\{n_{\mathrm{SU}}^{-i} \neq 0\right\}}\right) \cdot \mathbf{1}_{\left\{\sum_{k=1}^{N} n(k)=M, n_{\mathrm{SU}}^{-j}=0\right\}} .
\end{aligned}
$$

\section{Spectrum utilization}

We define the total spectrum or bandwidth utilization as follows

$$
U=\frac{1}{M} \sum_{\vec{n} \in S} \boldsymbol{P}(\vec{n}) \sum_{i=1}^{N} n(i),
$$

where $\boldsymbol{S}$ represent the space of states $(E$ or $F)$ and $\boldsymbol{P}(\vec{n})$ the steady state probability $(\pi(\vec{n})$ or $\bar{\pi}(\vec{n}))$ depending on the scheme.

\section{Numerical results}

We turn in this section to study a concrete example that will allow us to measure different metric performances of the system, and the various parameters that influence on that performances. Illustrative numerical examples are presented to demonstrate the interaction between the performance metrics and critical settings and to have a comparison with the theoretical analysis. We consider a system consisting of three WSPs $(N=3)$. The set of parameters used in this section is summarized in Table 2.

\section{Comparison and impact of our schemes}

In what follows we denote by $\mathbf{S c h} \mathbf{1}$ the no priority scheme and by Sch2 the priority one, and also we will refer in figures to our approximative method by (A) and the exact method by $(\mathbf{E})$. In all figures, the arrival rate is in (call/sec). We will start by studying the case of two WSPs $(N=2)$. $\lambda_{\mathrm{PU}}^{1}$ and $\lambda_{\mathrm{PU}}^{2}$ were obtained from Figure 2 that was used to develop a baseline to which SU improvements could be compared. Each service provider must deliver a certain level of QoS to its primary users based on the blocking probability $P_{B}(i, P U)$. It is then important to know the maximum PUs arrival rate that can be supported by the system while achieving this QoS. We plot in Figure 2 the blocking probability of primary users for the two schemes to show the maximum arrival rate supported while limiting blocking at $1 \%$. The result obtained is quite normal, sine the blocking probability of PUs depends only on the PUs arrival rate. We note that under this setting, the maximal arrival rate for PUs is $\lambda_{\mathrm{PU}}^{1}=1.2$ for the first WSP and $\lambda_{\mathrm{PU}}^{2}=0.5$ for the second. We'll use these values in the rest of numerical examples.

The difference between exact and approximative method, is that we consider that there is an independence between all WSPs in the system. Indeed, we considered this hypothesis based on the fact that each WSP is independent. This would mean that these service providers do not exchange their settings, nor reveal their resources for others. The difference between stationary distribution calculated from both approximative and exact method is about $2.10^{-4}$ in most cases, which is small enough to confirm that our main hypothesis reduces the calculating time considerably and helps to benefit from the reasons discussed above without changing the characteristics and the nature of the system. This small

Table 2 Main parameter values

\begin{tabular}{lccc}
\hline Parameters & WSP $\mathbf{1}$ & WSP 2 & WSP 3 \\
\hline $\mathrm{K}$ & 12 & 10 & 7 \\
$\mathrm{Th}$ & 7 & 5 & 3 \\
$\mu_{\mathrm{PU}}$ & 0.3 & 0.4 & 0.4 \\
$\mu_{\mathrm{SU}}$ & 0.3 & 0.4 & 0.4 \\
$\lambda_{\text {new }, \mathrm{SU}}$ & Variable & 0.4 & 0.3 \\
$\lambda_{\text {out }, \text { SU }}$ & 0.3 & 0.3 & 0.3 \\
\hline
\end{tabular}




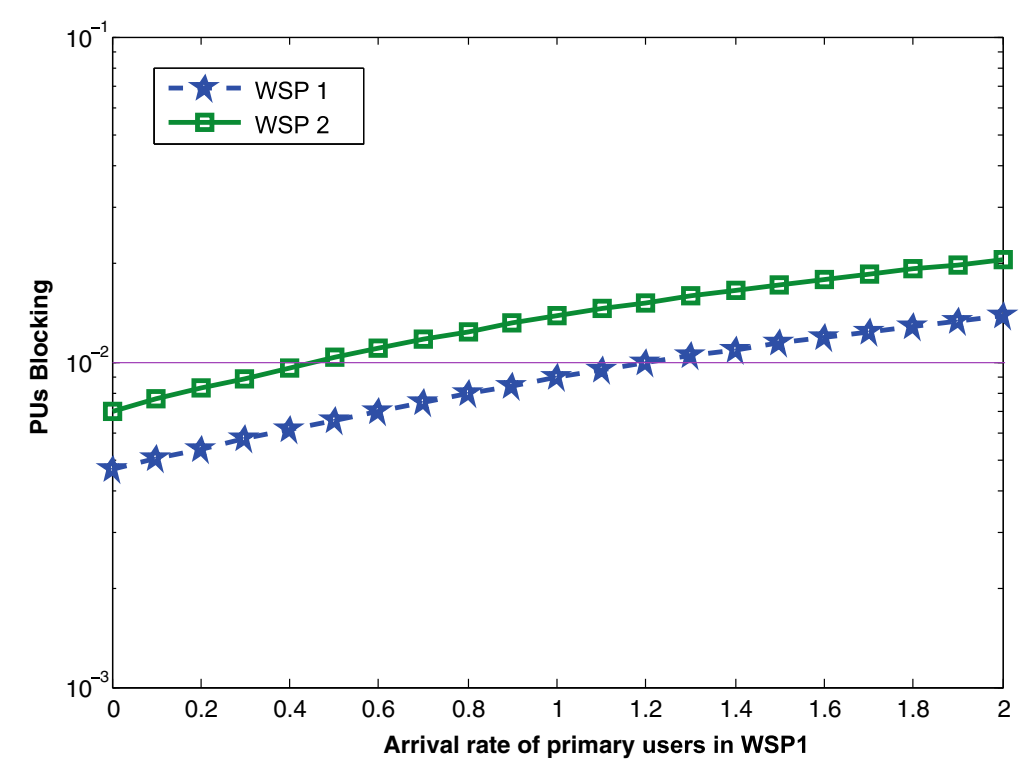

Figure 2 Blocking PUs. Blocking probabilities of PUs as a function of arrival rate.

variation between the two methods appears clearly in Figures 3 and 4.

In Figure 3, we depict SUs blocking probability as a function of arrival rate of SUs to the first WSP. When a new SU ask to have service in its home WSP, it has more chance with Sch2 to find either an empty channel or to drop an external SU. Unlike Sch1, where a new own SU looks only for an empty channel. Indeed, by applying priority we can improve performance and have a lower blocking probability than that obtained with the first scheme.
According to Figure 4 dropping probability was improved (i.e., decreased) from Sch1 to Sch2. Also in Sch2 the efficiency of priority appears clearly in the values of dropping. Effectively, for small and medium values of arrival rate $\left(\lambda_{\text {new } S U}^{1}\right)$, we notice that the dropping in the first WSP (Figure $4 \mathrm{a}, \mathrm{b}$ ) is lower than in the second one. However this behavior is not present in (Figure 4c). This is resulting from the politic followed in each scheme.

Indeed, in Sch1 the migration rate to the second WSP is high (Figure 5a), and this is resulting from the growth of the number of SUs that arrives to the first WSP and

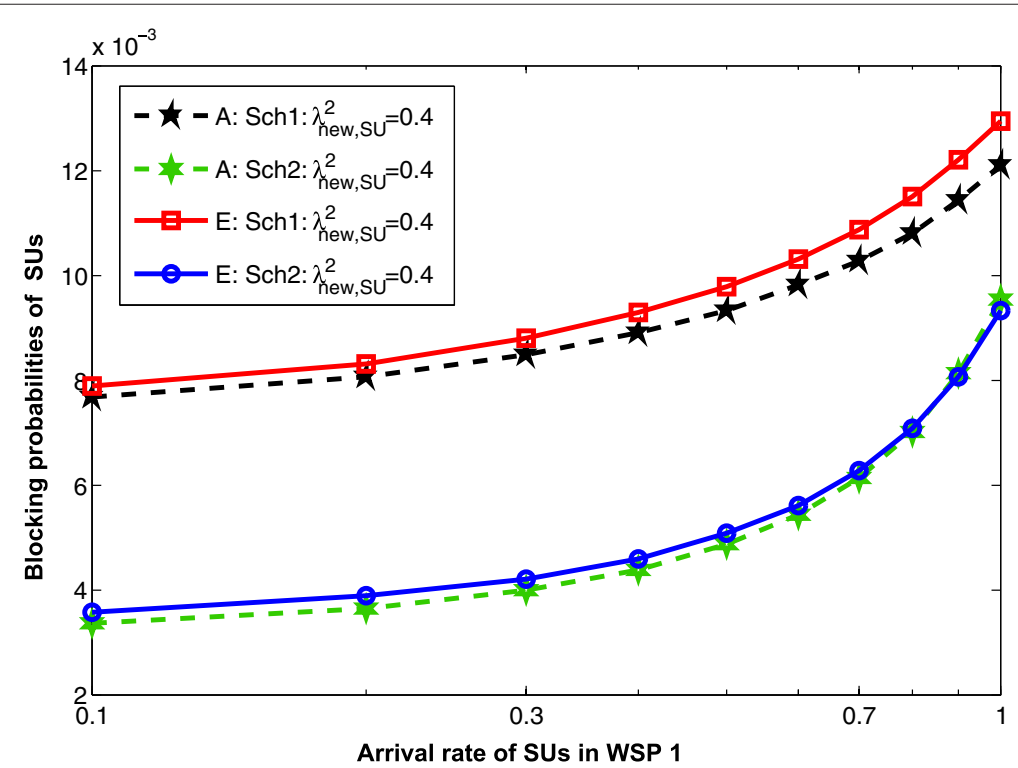

Figure 3 Blocking probabilities of SUs. Blocking probabilities of SUs-Varying the WSP 1 cognitive arrival rate for approximative and exact method. 


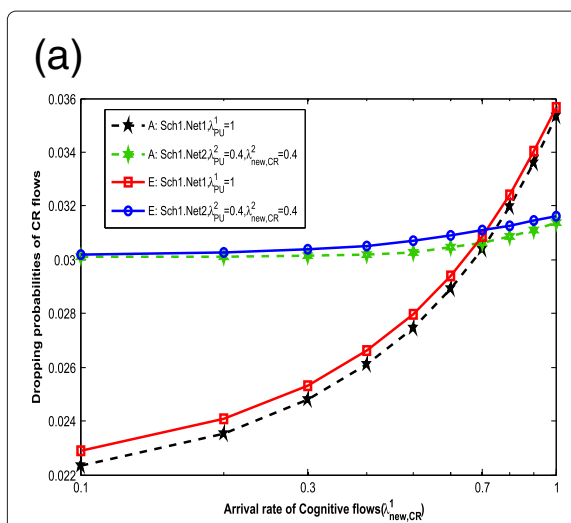

(b)

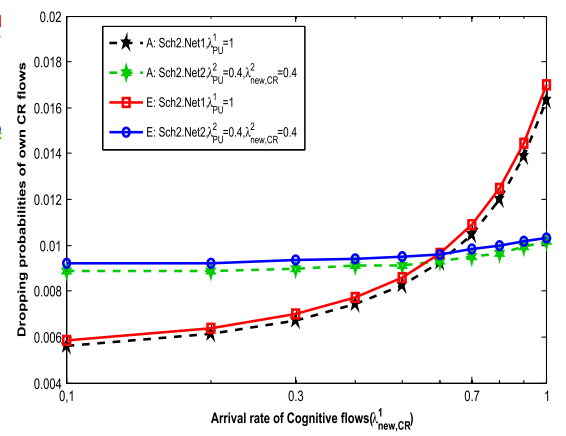

(c)

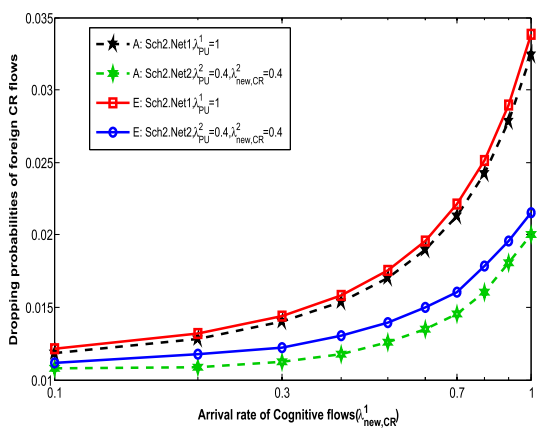

Figure 4 Dropping probabilities of SUs for the two schemes-Varying the WSP 1 cognitive arrival rate. (a) Dropping probabilities of SUs using Sch1. (b) Dropping probabilities of own SUs using Sch2. (c) Dropping probabilities of foreign SUs using Sch2.

consequently the occupation of all channels $K_{1}$. The saturation of WSP 1 generates more handover and more SUs in the second WSP and as a result more dropping. As for the Sch2, priority between SUs decreases considerably the values of migration rates (Figure $5 \mathrm{~b}$ ) (i.e., an incoming $\mathrm{SU}$ has a high probability to be served in his own WSP), but foreign SUs are more dropped in the first WSP because they are dropped by both primary $\left(\lambda_{\mathrm{PU}}^{1}=1.2\right)$ and own SUs.

However, own SUs are dropped more in the second WSP and it is related to the arrival rate of this kind of users $\left(\lambda_{\text {new,SU }}^{2}=0.4\right.$ and $\left.\lambda_{\text {new,SU }}^{1}<0.6\right)$ and also the number of channels available in each WSP. But we can remark that once $\lambda_{\text {new,SU }}^{1}$ exceeds the value of 0.6 (which represents the saturation value for $K_{1}=12$ channels), curves change and depends more on the arrival rate of PUs $\left(\lambda_{\mathrm{PU}}^{1}=1.2, \lambda_{\mathrm{PU}}^{2}=\right.$ $0.5)$. Priority levels show through values $(0.017$ as maximum value for own and 0.034 for foreign SUs in the first
WSP and 0.01 as maximum value for own and 0.021 for foreign in the second WSP, nearly 50\%) that users who use a part of their traffic in a cognitive manner (when their demand are not delay-sensitive) have less risk to be dropped before they finish their service comparing to external users.

Improving spectrum utilization is an important task for enhancing and sustaining the growth of mobile users with limited spectrum. We plot in Figure 6 the spectrum utilization for both schemes. For low traffic (small values of cognitive arrival rate), applying priority between own and foreign SUs can antagonize and penalize spectrum utilization. That's why, it's better for a service provider to apply the Sch1 in this situation. But, when arrival rate of SUs starts to grow (medium and high traffic) priority scheme works better and improves more the spectrum utilization. This result is coming from the behavior of the three kind of users. own (a)

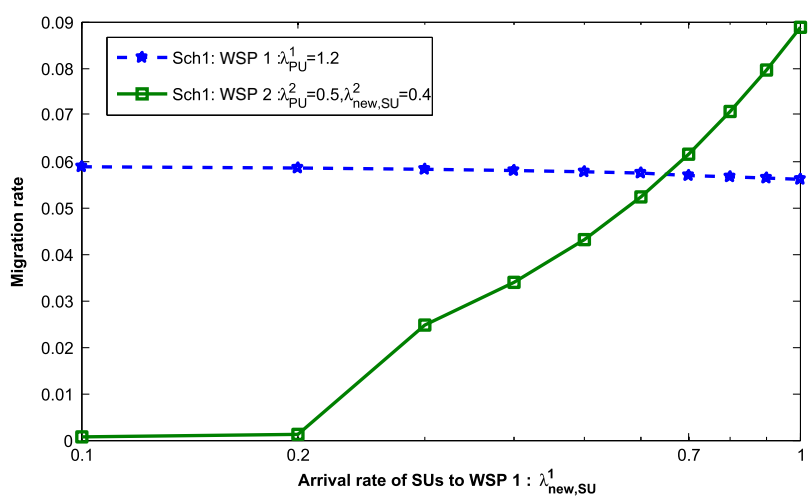

(b)

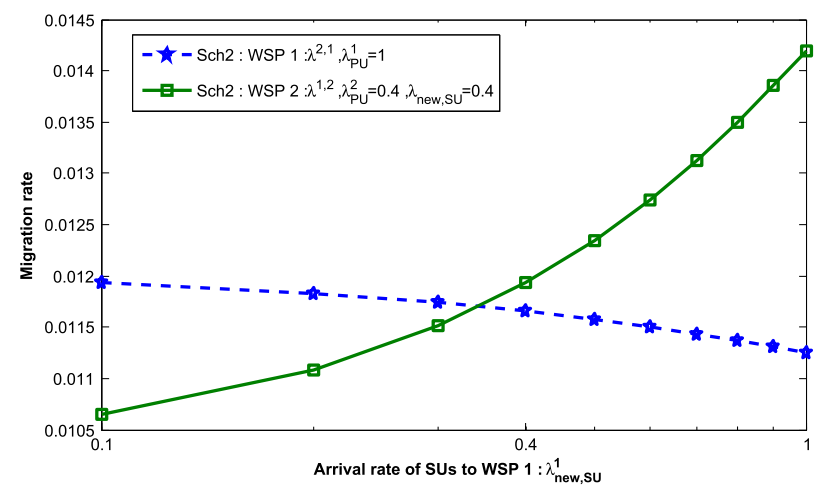

Figure 5 Migration rate. Migration rate to each WSP for the two schemes-Varying the WSP 1 cognitive arrival rate. 


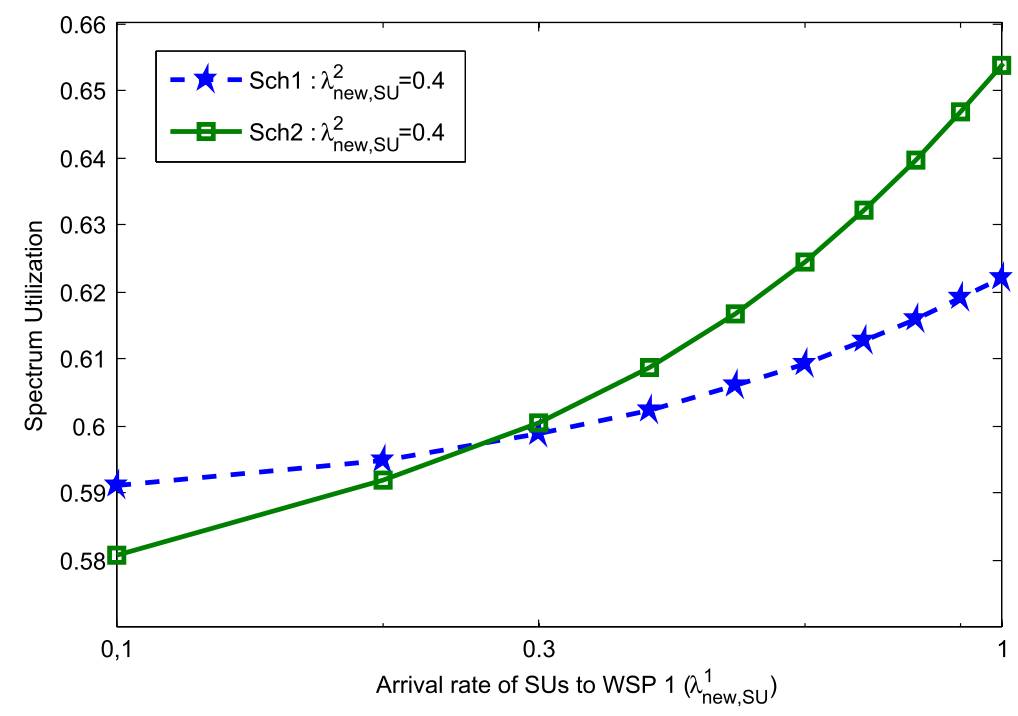

Figure 6 Spectrum utilization. Spectrum utilization-Varying the WSP 1 cognitive arrival rate in call/sec.

SUs are less blocked and dropped in the Sch2, which results more spectrum utilization and more time using the system.

\section{Using approximative solution with $\mathrm{N}=3$}

In this section, we examine the case where the number of WSPs is more than $2(N>2)$. We recall that the exact solution takes more time and becomes more complex when $N$ increases. For this reason, we use our approximative solution in the next simulations when $N=3$. In Figure 7, we plot the blocking probability of SUs for the two schemes. The results show that even with multiple WSPs, the priority scheme still offer less blocking for own SUs for the same reasons discussed before.

We can make the same remark for the case of dropping probability illustrated in Figure 8. For small values of $\lambda_{\text {new,SU }}^{1}$ the dropping in WSP 2 and 3 is constant, and starts growing with the increasing of the arrival rate of

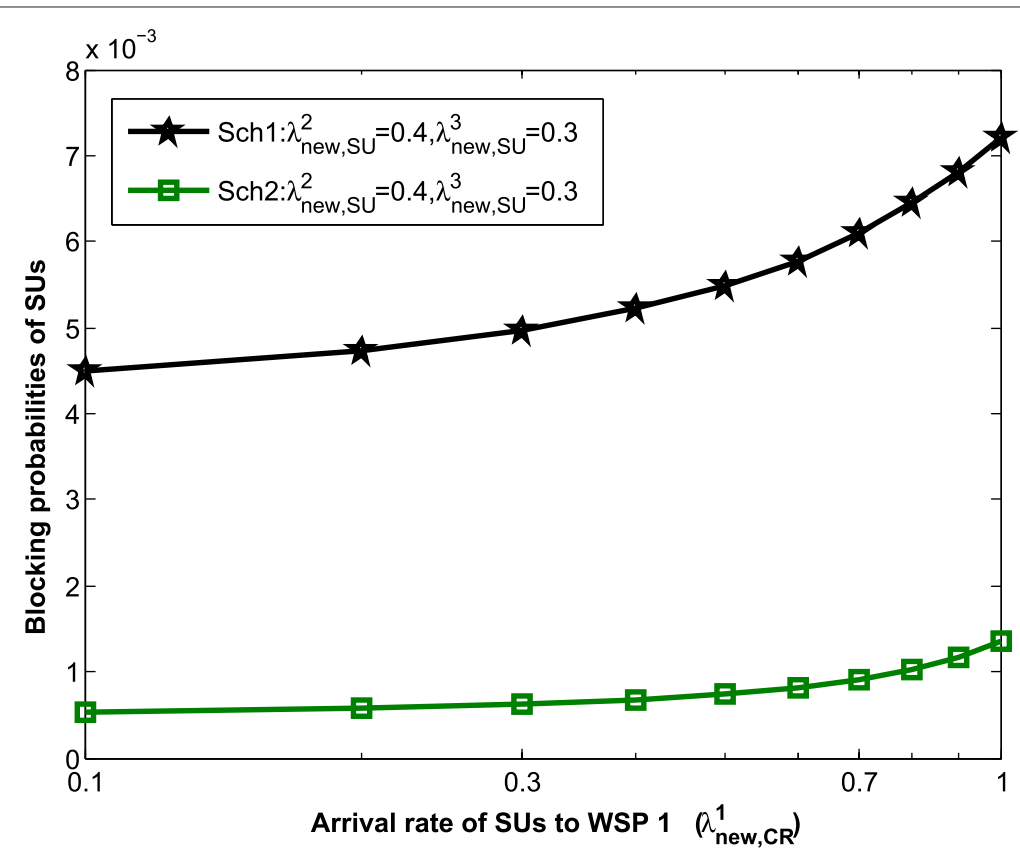

Figure 7 Blocking probabilities of SUs. Blocking probabilities of SUs-Varying WSP 1 cognitive arrival rate (call/sec) for approximative solution with 3 WSPS. 


\section{(a)}

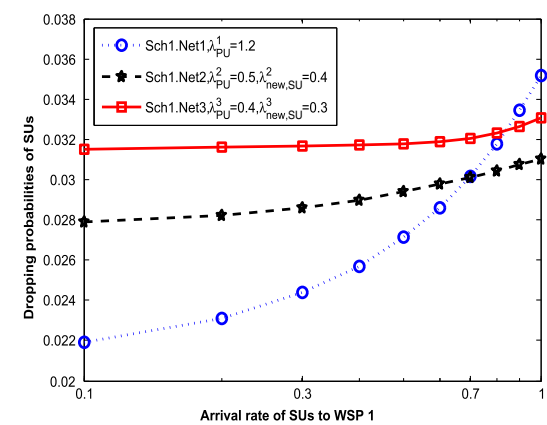

(b)

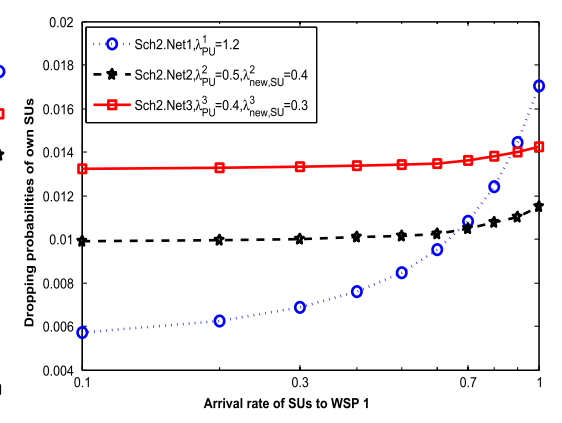

(c)

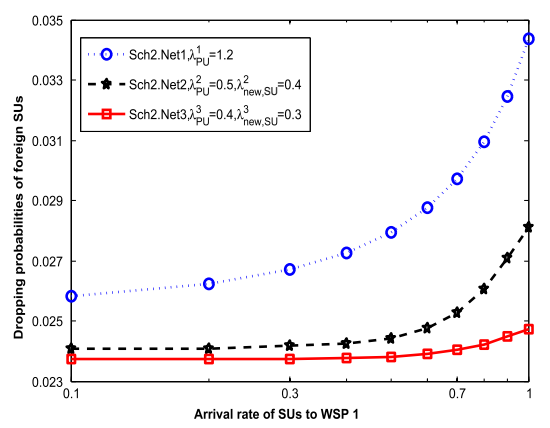

Figure 8 Dropping probabilities of SUs for the two schemes-Varying the WSP 1 cognitive arrival rate in call/sec in a system with 3 WSPs. (a) Dropping probabilities of SUs. (b) Dropping probabilities of own SUs. (c) Dropping probabilities of foreign SUs.

SUs. Indeed, the migration from WSP 1 to the others starts after saturation. Also, foreign SUs are less dropped in small WSPs (Figure 8c) because the rate of migration is low.

\section{Conclusion and discussions}

In this article, we have studied the QoS and the performance evaluation in wireless networks. The system proposed here is a set of wireless service providers. We define three different kinds of users: primary users with dedicated channels, mixed users that have the possibility to split their traffic into real time and best effort traffic and finally SUs who sense the channels for spectrum holes.

Moreover, a dynamic access control for SUs was proposed for two schemes. Indeed, we propose a scheme where the access control is related to the total number of SUs present in the system (no priority between them), and to the threshold fixed by the WSP. While in the second scheme, more priority is given to what we called "own SUs". In fact, own SUs could be dropped in their home WSP only by PUs. Whereas foreign SUs could be dropped by both PUs and own SUs of each WSP. Furthermore, we have seen that improving the service time of SUs is possible with respect to the QoS offered to PUs.

The model developed describes the interaction between users in one side, and between users and service providers in the other side. The system is modeled by a Markov process, with continuous time and finite state space. Using this Markov process, we have calculated the exact solution and proposed an approximation to make the problem much easier to solve, without changing its characteristics. Moreover, the application of this approximation is more realistic in this context, since a service provider has only partial information about the system. We show numerically that the two solutions are too close from each other with a negligible difference.

In addition, several numerical results are presented in this article in order to analyze the two schemes of our study. Through these experiments, it has been verified that priority levels applied to SUs allows an improvement in QoS or the support of additional best effort users without deteriorating the QoS of PUs. We believe that how a service provider should choose the threshold is a crucial task. Indeed, decreasing the threshold could produce a high migration rate of SUs to other WSPs and brings down their performance. The problem becomes one of noncooperative games and could be the extension of this article in the future.

\section{Competing interests}

The authors declare that they have no competing interests.

\section{Acknowledgements}

A part of this article was presented in the 13th ACM International Conference on Modeling, Analysis and Simulation of Wireless and Mobile Systems,

October 17-21, 2010 Bodrum, Turkey.

\section{Author details}

${ }^{1}$ LIA/CERI, University of Avignon, Agroparc BP 1228, Avignon, France. ${ }^{2}$ LIM, Sidi Mohamed Ben Abdellah University, BP 1796, Fez, Morocco. ${ }^{3}$ LARIT/FSK, Ibn Tofail University, BP 133, Knitra 14000, Morocco. ${ }^{4}$ LIMIARF/FSR, University of Mohammed V-Agdal, BP 1014 RP, Rabat, Morocco.

Received: 6 February 2012 Accepted: 6 August 2012

Published: 18 September 2012

\section{References}

1. A Sahai, R Tandra, SM Mishra, N Hoven, Fundamental design tradeoffs in cognitive radio systems. in In Proceedings of the first international workshop on Technology and policy for accessing spectrum (TAPAS '06) (ACM, New York, 2006), Article 2

2. J Mitola III, Cognitive radio: an integrated agent architecture for software defined radio. PhD thesis, KTH Royal Institute of Technology, Stockholm, Sweden, (2000)

3. J Mitola III, Cognitive radio for flexible mobile multimedia communications. Mob. Netw. Appl. 5(6), 435-441 (2001)

4. Y Zhang, Dynamic spectrum access in cognitive radio wireless networks. in Proceedings of International Conference on Communications (IEEE ICC 2008), Beijing, China, May 19-23, 2008, pp. 4927-4932

5. D Cabric, SM Mishra, RW Brodersen, A Wolisz, D Willkomm, White paper: corvus: a cognitive radio approach for usage of virtual unlicensed spectrum. [online]. available: http://bwrc.eecs.berkeley.edu/research/ mcma/crwhitepaperfinal1.pdf 
6. V Kone, L Yang, X Yang, BY Zhao, $\mathrm{H}$ Zheng, The effectiveness of opportunistic spectrum access: a measurement study. IEEE/ACM Trans. on Networking. (99), 1 (2012)

7. M Zhang, Performance analysis of the cognitive radio network with a call level queue for secondary users. in Proceedings of International Conference on Wireless Communications, Networking and Mobile Computing (IEEE WiCOMO9) IEEE. Beijing, (2009), pp. 1-4

8. O Ali Ercan, J Lee, S Pollin, JM Rabaey, A revenue enhancing stackelberg game for owners in opportunistic spectrum access. in Proceedings of Dynamic Spectrum Access Networks (DySPAN08) (Chicago, Illinois, 2008)

9. Y-C Liang, Y Zeng, ECY Peh, AT Hoang, Sensing-throughput tradeoff for cognitive radio networks. IEEE Trans. Wirel. Commun. 7(4), 1326-1337 (2008)

10. YH Zeng, Y-C Liang, AT Hoang, R Zhang, A review on spectrum sensing for cognitive radio: challenges and solutions. EURASIP J. Adv. Signal Process. 2010, (2010)

11. Z Quan, S Cui, A Sayed, Optimal linear cooperation for spectrum sensing in cognitive radio networks. IEEE J. Sel. Top. Signal Process. 2(1), 28-40 (2008)

12. B Zhao, S Shimamoto, Energy-aware cooperative spectrum sensing with inter-channel interference cancellation for cognitive radio networks. in Proceedings of 20th International Conference on Computer Communications and Networks (ICCCN) (2011), pp. 1-6

13. HB Salameh, M Krunz, D Manzi, An efficient guard-band-aware multichannel spectrum sharing mechanism for dynamic access networks. in IEEE Global Telecommunications Conference (GLOBECOM) (2011), pp. 1-5

14. E Jung, X Liu, Opportunistic spectrum access in multiple-primary-user environments under the packet collision constraint. IEEE/ACM Trans. Network. 20(2), 501-514 (2012)

15. SM Mishra, RTandra, A Sahai What is a spectrum hole and what does it take to recognize one? Proc. IEEE. 97(5), 824-848 (2009)

16. H Kim, CT Chou, KG Shin, What and how much to gain from spectrum agility. in Proceedings of Real-Time Comput. Lab., Univ. Michigan, Ann Arbor, MI, Tech. Rep. (2005)

17. B Ishibashi, N Bouabdallah, R Boutaba, Qos performance analysis of cognitive radio-based virtual wireless networks. in Proceedings of The 27th IEEE Conference on Computer Communications (INFOCOM) Phoenix, USA, (2008), pp. 2423-2431

18. K Ibrahimi, R Elazouzi, SK Samanta, EH Bouyakhf, Adaptive modulation and coding scheme with intra- and inter-cell mobility for hsdpa system. in Proceedings of the Sixth International Conference on Broadband Communications, Networks, and Systems (IEEE/ICST BROADNETS09) (Madrid, Spain, 2009)

19. D Niyato, E Hossain, Call admission control for qos provisioning in $4 \mathrm{~g}$ wireless networks: issues and approaches. IEEE Network. 19(5), 5-11 (2005)

20. R Cooper, Introduction to Queueing Theory (North-Holland, New York, 1981)

\section{doi:10.1186/1687-1499-2012-296}

Cite this article as: Raiss-El-Fenni et al:: Dynamic spectrum allocation with admission control based on cognitive radio for QoS support in multiple wireless network. EURASIP Journal on Wireless Communications and Networking 2012 2012:296.

\section{Submit your manuscript to a SpringerOpen ${ }^{\circ}$ journal and benefit from: \\ - Convenient online submission \\ - Rigorous peer review \\ - Immediate publication on acceptance \\ - Open access: articles freely available online \\ - High visibility within the field \\ - Retaining the copyright to your article \\ Submit your next manuscript at $\boldsymbol{\nabla}$ springeropen.com}

